\title{
Convalescent Plasma Therapy and its Century-Old Untapped Potential for COVID-19
}

\author{
Samahir Tariq Khan ${ }^{\text {a }}$, Sajjad Ali ${ }^{\text {a*}}$, Nisha Lohana ${ }^{\text {a }}$ \\ ${ }^{a}$ Ziauddin Medical University, Karachi, Pakistan.
}

Received 24 August 2020; Accepted 28 October 2020

\begin{abstract}
Background: The COVID-19 virus, in terms of pathogenesis and disease spectrum, resembles its predecessor viral strains which caused outbreaks of SARS and MERs. Due to unavailability of approved treatment protocols, healthcare workers initiated treatment of COVID-19 patients with convalescent plasma therapy. Objective: To appraise similarities between the three Coronaviruses and deduce the effectiveness of CP therapy based on exploration of its efficacy in the SARS and MERS outbreaks. Analysis: A narrative review of case reports, randomised controlled trials and meta-analysis studies, on use of CP therapy in SARs and MERS, was conducted. Studies evaluated for the purpose of this review were added through search engines of PubMed Central and Google Scholar. Results: We concluded that CP therapy had been able to play pivotal roles in treating critically-ill SARS and MERS patients. The risks of unintended immunological responses among other factors, hindering CP Therapy's approval from drug administration authorities, were weighed against favourable chanisms, such as hypercoagulability, that support its use in COVID-19 patients. Conclusion: Findings collected from these studies steered our path to theorize the possibility of reducing mortality with convalescent plasma therapy and support our rallying efforts for enlisting this in the official treatment protocol of COVID-19.
\end{abstract}

Keywords: COVID-19; Convalescent Plasma Therapy; Transfusion; Coronavirus; Microbiology.

\section{Introduction}

Coronaviridae are single-stranded, enveloped RNA (ribonucleic acid) viruses, betacoronavirus being the prime talk in the field of medicine [1]. Human and animal variants of the betacoronavirus are largely associated with mild respiratory symptoms, whereby both human-to-animal or animal-to-animal transmissions have been consistently identified. In the past, however, two of these pathogens were known to cause highly transmissible respiratory illnesses that led to alarming regional outbreaks, namely SARS-CoV and MERS-CoV [2].

The current Public Health Emergency of International concern, as the World Health Organization (WHO) quotes, was set in motion after a pneumonia-like illness first appeared in Wuhan, China. Extensive research using deep sequencing techniques and clinical reports gathered from healthcare workers globally, the disease now labelled COVID-19, is caused by a species of coronavirus with a higher infectivity rate than previous outbreaks [3]. On $11^{\text {th }}$ March, approximately three months after the origin, WHO characterized COVID-19 as a pandemic. This highly communicable disease has since infected 2,436,743 people worldwide and caused 165,310 deaths (latest update as of $22^{\text {nd }}$ April 2020). As of now, 213 countries or territories have been infected with this deadly virus, while studies suggest close proximity with infected patients as the major source of disease spread [4].

Repeated outbreaks and emergence of new species foreshadow the crippling status of our healthcare system and failure of cutting-edge research tactics, as no preventive or therapeutic measure is currently available for either virus

* Corresponding author: sajjad110@live.com

\section{doi http://dx.doi.org/10.28991/SciMedJ-2020-0204-6}

$>$ This is an open access article under the CC-BY license (https://creativecommons.org/licenses/by/4.0/).

(C) Authors retain all copyrights. 
species. Lately, medical professionals around the globe are considering opting for conventional treatment methods for COVID-19, i.e. convalescent blood plasma therapy, which was successfully implemented on other variants of coronavirus such as SARS and MERS. In this article, we review this potential technique as adjunctive treatment for newly emerging diseases, deemed partially successful in the past.

\section{Fundamentals of Convalescent Plasma Therapy}

Implementation of Passive immunization (PI) for both prevention and treatment of emerging infectious diseases can be traced back to the early 20th century. This is a simple method to achieve rapid short-term immunization, by delivering pathogen-specific antibodies to infected patients. Initially obtained from animal sera (mostly horses and rabbits) this concept has been a lifesaver for many acute infections since its introduction and has shown promising roles in cancer immunotherapy [5, 6]. Human blood or serum was in contemplation but not widely used. Although antibiotics have superseded this age-old technique for treatment of bacterial infections, it remains a potent tool for viral infections wherein lack of vaccines or treatment is noted $[6,7]$.

Convalescent blood products (CBP) are a potential source of antibodies originating from immune cells of a patient after being exposed and surviving a certain infectious disease. This newly gained humoral immunity can be extracted via CBP and later transfused into patients with similar illness. CBP transfusion can neutralize the pathogen and ultimately contribute to its complete removal from an infected patient's blood circulation [8, 9]. The following CBPs have been in use, as suggested by studies: (i) convalescent whole blood (CWB), convalescent plasma (CP) or convalescent serum (CS); (ii) pooled human immunoglobulin (Ig) for intravenous or intramuscular route of administration; (iii) high-titre human Ig; and (iv) polyclonal or monoclonal antibodies [5-8]. Separation of blood into its components for passive immunity involves apheresis which involves taking whole blood from a donor (patient), separating its different components and extracting one specific component [10]. The remaining (non-usable) blood components are reinserted into the donor's (patient) bloodstream. Using this technique to withdraw plasma is of great advantage as ample amounts of plasma can be extracted in each session and no known adverse effects on the donor's hemoglobin, due to re-infusion of its red blood cells, are noted [11]. This can further be illustrated in Figure 1 [12].

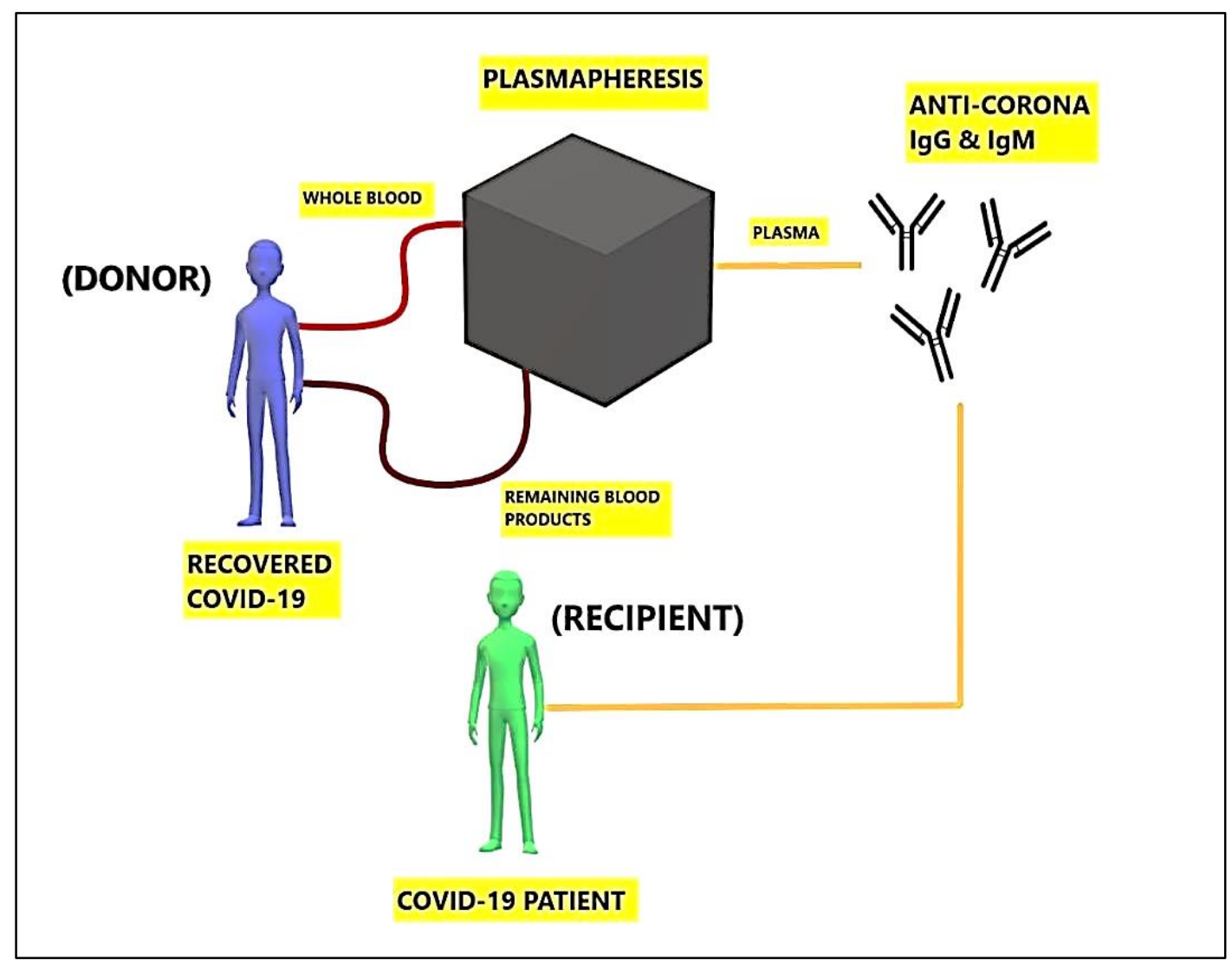

Figure 1. Fundamentals of convalescent plasma therapy

\section{Advancements in Convalescent Blood or Plasma Therapy Since 1890}

The first approach of using CP therapy dates back to 1890 when blood serum was used for the treatment of diphtheria. Initially obtained from immunized animals, the discovery of human blood or serum containing potential antibodies mimicking those found in animal sera, was later made. Some other examples of bacterial diseases wherein 
CP was utilized are scarlet fever and pertussis, in the 1920s and the 1970s respectively [11]. Studies conducted in the year 1918 [13], amidst the Spanish influenza pandemic, demonstrated effective use of CBP, including a meta-analysis which showed substantially reduced mortality risk in patients treated with CBP [14-19]. The positive response of CP with reference to the recently, avian $\mathrm{H} 1 \mathrm{~N} 1, \mathrm{H} 5 \mathrm{~N} 1$ and other organism related acute respiratory infections (SARI) was evaluated based on clinical applications on patients, in the coming decades. In addition, animal models of influenza pneumonia have also shown satisfactory response of convalescent serum [20-30]. Other viral infections [31-33] such as Influenza [34], mumps [35], Herpes zoster [36], Poliomyelitis [37], and measles were also treated effectively [29, 38]. However, in some of those early clinical examples, whole blood was transformed into serum by allowing it to clot, specifically known for treatment of measles [29].

Regarding the potency of CP for use in haemorrhagic fever diagnosis, a young woman with Ebola Virus (EBOV) was transfused plasma from a survivor of Marburg virus, a closely related infection.

This however, reaped no benefits. Although WHO prioritized the use of CP for Ebola Virus Disease (EVD) during the 2014 outbreak $[39,40]$, a study showed inadequate response to survival rates where CP was used [41]. Contrarily, patients with Argentine haemorrhagic fever, receiving CP treatment [42-45] showed lower mortality rates in a double blind study conducted in 1979 compared to subjects treated with "natural plasma". Another study on this, stretching over approximately three decades, showed a substantial difference in total mortality among patients diagnosed with traditional or CP care. Likewise, patients diagnosed with Lassa fever, another type of viral haemorrhagic fever, apparently showed reassuring results after receiving CP [46]. Despite procedural limitations and insufficient statistics, reference from above cited studies corresponds to the use of CP for various infections known [47].

\section{SARS-CoV \& Use of Convalescent Plasma}

Close to comparison with COVID-19 is SARS or Severe Acute Respiratory Syndrome (SARS), which surfaced in Guangdong Province, China in the fall of 2002 and spread to 29 territories causing about 8422 infected cases and 916 deaths. SARS alarmed the world of its first ever pandemic threat of the new century [48, 49]. Following an unparalleled global public health campaign, the epidemic was contained within seven months of its first occurrence [50].

A study by Cheng et al. (2005) reported CP transfusion to 80 SARS patients, two weeks following the onset of their symptoms, along with antiviral ribavirin and methylprednisolone, between 20 March 2003 and 26 May 2003 . No significant side effects were recorded however patients transfused on day 14 displayed a faster discharge rate in comparison to those who received $\mathrm{CP}$ on day 22. This implies that $\mathrm{CP}$ administered earlier (that is, prior to 14 days of disease starting) during the course of the disease, might prove to be more favourable [51].

In an unrelated study by Yeh et al. (2005) CP obtained from healthcare workers exposed to SARS patients, after failure with ribavirin and methylprednisolone, was administered before day 14 of the illness. Subsequent documentation of anti-SARS IgM and IgG and other findings revealed that the virus was cleared within a day of transfusion, followed by subsidence of fever and resolution of pulmonary infiltrates [52].

A case study on a 57-year-old SARS patient, receiving ribavirin and prednisolone showed positive progress with reduced viral load. On day 14 she was administered CP as adjunctive treatment and within a few days fever subsided and chest X-ray showed further resolution of basal lung infiltrates. Furthermore, Lingui Kong wrote in his report about a 28 -year-old pregnant female, clinically worsening, with steroids, antiviral medications and a respirator all deemed ineffective [53]. However, impressive recovery occurred within 12-36 hours of CP transfusion. An interventional study carried out in the Hong Kong Chinese University, 40 patients were randomly divided into a control group and an experiment group (those transfused with CP) [54]. The result was insufficient because of small sample size but satisfactory results were observed in those with $\mathrm{CP}$ intervention. Based on a multi-research conclusion, use of convalescent plasma within the first two weeks might help shorten long hospital stays and adequately reduce mortality [55].

\section{Evidence-based Deductions of MERS-COV Management with Convalescent Plasma}

In the heart of the Arabian Peninsula, emerged MERS-CoV, a respiratory illness causing species of coronavirus in 2012 in Saudi Arabia [56]. Named as the Middle Eastern Respiratory syndrome Coronavirus, outbreaks were reported in regions as far as East Asia. As of January 2020, WHO reported a total of 2519 laboratory-confirmed cases, including 866 associated deaths (case fatality rate: 34.3\%) [57]. Since no targeted medication and vaccine was available, global search for a standard therapeutic protocol was underway. With a symptom streak very similar to COVID-19, we hope to find evidence-based deductions of using Convalescent Plasma therapy to treat and manage patients marked positive in the current COVID-19 pandemic.

As mentioned earlier, passive immunotherapy with neutralizing antibodies was a heavily-used tactic to combat the SARS epidemic. Hence, treating severely-ill MERS-CoV with CP was a go-to treatment option for healthcare 
workers. Chan et al. (2013) produced his study, within a year of the outbreak, calling attention to the use of previously collected sera from SARS patients for it contained cross-reactive neutralizing antibodies which also proved to work on this novel species [58]. Several other researches directed against finding the transmission source of the virus, used serum samples from dromedary camels in the Middle East. These identified profound levels of MERS-CoV-specific antibodies, further strengthening the dire need of putting the CP theory into application [59-61].

A study identifying these antibodies by Corti et al. (2015) isolated a potent MERS-CoV-neutralizing antibody (LCA60) from memory B cells of an infected individual, which could neutralize MERSCoV [60]. The International Severe Acute Respiratory and Emerging Infection Consortium (ISARIC) presented its recommendations on clinical decision-making support for clinicians. This was last revised in 2017 and highlights facts pertaining to similarities between SARS-CoV and MERS-CoV, thus providing benefits of adapting a similar treatment route [62].

Furthermore, a case report from a tertiary care hospital in Republic of Korea outlined the progress of 3 patients with respiratory failure out of a total 45 MERS-CoV positive cases admitted. Positive MERS-CoV patients with asymptomatic or mild disease processes were contacted in the $3^{\text {rd }}$ week of their illness to kick-off CP therapy. This time frame was chosen on the basis of presumed seroconversion rates that show maximum response in the 3rd week [63].

Plasma drawn from the donors was checked for MERS-CoV PCR titers and any blood-borne pathogens, HBV, HCV, HIV and syphilis, namely [64]. Patients' progress was closely monitored with serological markers once or twice after the infusion. The quantitative values of Anti-MERSCoV ELISA IgG, ELISA IgA and indirect fluorescent antibody (IFA) IgM along with MERS-CoV plaque reduction neutralization test (PRNT), were then matched against cut-off values discovered in the study of Ko et al. (2016) [65]. The role of seroconversion was further highlighted in another study whereby serologic responses were evaluated in 42 MERS patients who were grouped according to disease severity [66]. Highest seroconversion was observed in patients with pneumonia withor without respiratory failure. The results led us to the conclusion that plasma be collected from donors with moderate to severe respiratory illness for optimum antibody activity and neutralizing activity of a PRNT titer $\geq 1: 80$, to carry out effective CP infusion. ELISA IgG could substitute for the neutralization test in resource-limited situations [63].

A detailed research by Arabi et al. (2016) screened serum samples to make an advanced understanding of the feasibility of using convalescent plasma immunotherapy against MERS-CoV infection. With a sample size of 443 plasma donors, including healthcare workers and household contacts of laboratory-confirmed patients, the findings paved the way for a successful clinical trial in future. But extensive serum screening would be required due to low probability of donors with sufficiently high antibody titers. Antibody reactivity was found to be associated with the timing of sample collection and the severity of illness. However, low seroreactivity results hinted at a possibility of short antibody responses, which should be further looked into. Furthermore, screening of convalescent plasma for presence of MERS-CoV antibodies was proved to be an indispensable tool owing to low prevalence of antibodies calculated [67].

Concluding the study-based evaluation of convalescent plasma therapy for MERS-CoV, we shall quote the exploratory meta-analysis namely, The Effectiveness of Convalescent Plasma and Hyperimmune Immunoglobulin for the Treatment of Severe Acute Respiratory Infections of Viral Etiology. Though the essence of this review revolved around SARS-CoV infection, the inference drawn supported the possibility of using convalescent plasma for MERS$\mathrm{CoV}$ with a well-devised clinical trial taking place beforehand [68].

\section{Using Convalescent Plasma in the Current COVID-19 Pandemic}

In the past, WHO through the Blood Regulator Network has recommended the utilization of convalescing plasma or serum as empiric therapy when both vaccination and medication is unavailable for a certain infection [73]. Nonetheless, as of now, WHO guidelines concerning COVID-19 management to target prevention, case detection and monitoring, and supportive care alongside simultaneous contact tracing [74].

The first use of CP for COVID-19 was reported in China, by Shen et al., when 5 of the most critically ill patients were transfused with antibody-containing plasma. Patients with no comorbidity received plasma 10 days ahead of the patient with known chronic disease. After confirmation of presence of IgG and IgM anti-SARS-CoV-19 antibodies and neutralization in in vitro cultures. While these patients continued with their specific antiviral treatment primarily with lopinavir / ritonavir and interferon, the use of convalescent plasma may have helped in rehabilitation, as all patients improved clinically within a week of the transfusion, in terms of organ failure assessment and body temperature regulation. The study was carried out from January 2020 to March 2020.

Another study, involving a batch of 245 COVID-19 positive patients received convalescent plasma injections, and improved clinical conditions were noted in 91 cases. All plasma used for transfusion had previously been checked for neutralizing antibodies [75]. 
A pilot study with 10 severely ill COVID-19 patients underwent plasma therapy as an adjunct to their antiviral drug combination. 10 randomly-selected patients from an ongoing cohort study, were used to analyze the different clinical outcomes of the two groups. All clinical symptoms substantially improved within 1-3 days of transfusion. After administering CP, three patients were weaned off from artificial ventilation to a high-fluid nasal cannula. In comparison, constant oxygenation was changed to sporadic in one patient. Chest CTs being the definite test of evaluation, demonstrated varying resorption of pulmonary lesions after CP transfusion in all these patients, who were previously reported to have massive infiltration and widespread ground-glass attenuation. Seven patients from the $\mathrm{CP}$ group being treated and discharged, meanwhile three patients from the control group succumbed. The findings also revealed that the $\mathrm{CP}$-contained antibodies had alleviated inflammation and overreaction of the immune system [76].

On April 13th US Food and Drug Administration published its recommendations for use of plasma in management of COVID-19 [77]. With components of the guideline highlighting issues related to its use, FDA has also stated that plasma from those recovering from covid-19 may contain virus antibodies may be effective to counter infection and plasma from recovered patients must be obtained who have shown no such symptoms for two weeks and have been tested negative for COVID-19. To be eligible for convalescent plasma treatment, FDA has announced that COVID-19 patients must have a severe or life threatening condition such as respiratory failure, septic shock, multi-organ dysfunction, shortness of breath and so on. Based on our preliminary findings, CP therapy for extreme COVID-19 patients can be a readily accessible, promising and safe rescue option.

\section{Potential Risks and Benefits Involved in Using Convalescent Plasma Therapy}

According to data included, the potential risks of passive convalescent sera administration are broadly divided into established and hypothetical. Established threats are blood circulation threats, including unintended contact with another infectious disease agent and serum-based reactions i.e. immunological responses, most commonly serum sickness. Chances of accidentally spreading recognized infective agents or inducing transfusion reactions, is small with current blood banking strategies and regular pathogen screening. On the other hand, hypothetical vulnerability involves an antimicrobial dependent enhancement (ADE) phenomenon, which is associated with an increase in the presence of certain antibodies. Laboratory studies have shown prospective use of one anti-coronavirus antibody for another strain, owing to the numerous pathways identified [68].

Surprisingly, convalescent plasma is also known to regenerate coagulation factors, along with inhibition of viral replication, in hemorrhagic fevers such as Ebola [69-71]. Inhibition of viremia, noted maximum in the first week of infection, is a pivotal parameter demonstrating the efficacy of CP therapy. Over the course of 10 to 14 days, a primary immune response accompanied by removal of the virus occurred. A study in vivo also found antibodies to impact free viral clearance and blocking of infection along with increased cell clearance. In closing, this entails that CP therapy administered at an initial stage of disease is more favorable [72].

\section{Conclusion}

Given the economic and thereby the healthcare system in underdeveloped and developing countries, where more than half the population cannot afford long and complex hospital stays or lockdowns for long stretches, the disease spread must be delayed at all costs. With evidence, we can conclude that localized herd immunity can help slow down its soaring infectivity rates in vulnerable populations, in the absence of an approved vaccine or successful drug regime. Convalescent plasma therapy could prove to be the most vital arsenal in the war against COVID19, with respect to its fairly-promising aforementioned results. Prompt action must be taken to identify all prerequisites required for this, along with appropriate testing of the plasma from recovered patients. Availability of this plasma ready-for-use can be proceeded with timely contact tracing, collection, antibody-testing and distribution where needed. Policy-makers with the help of medical personnel, working diligently on the forefront should, therefore, consider passive immunity as the mainstay for COVID-19 to protect the health of those most vulnerable i.e. immunocompromised patients, including the elderly and those with chronic diseases.

\section{Acknowledgements}

We gratefully acknowledge all the participants who participated in this study.

\section{Declaration of Competing Interest}

The authors declare that they have no known competing financial interests or personal relationships that could have appeared to influence the work reported in this paper.

\section{Ethical Approval}

The manuscript does not contain experiments on animals and humans; hence ethical permission not required. 


\section{References}

[1] Fehr, A. R., \& Perlman, S. (2015). Coronaviruses: An Overview of Their Replication and Pathogenesis. Methods Mol Biol, $1282,1$.

[2] Lu, R., Zhao, X., Li, J., Niu, P., Yang, B., Wu, H., . . Zhu, N. (2020). Genomic characterisation and epidemiology of 2019 novel coronavirus: implications for virus origins and receptor binding. Lancet, 395(10224), 565-574.

[3] Chen, Y., Liu, Q., \& Guo, D. (2020). Emerging coronaviruses: genome structure, replication, and pathogenesis. J Med Virol, 92(4), 418-423.

[4] Organization, W. H. (2020c, 2020/5/7). WHO Coronavirus Disease (COVID-19) Dashboard. Available online: https://covid19.who.int/

[5] Keller, M. A., \& Stiehm, E. R. (2000). Passive immunity in prevention and treatment of infectious diseases. Clin Microbiol Rev, 13(4), 602-614.

[6] Rajam, G., Sampson, J., M Carlone, G., \& W Ades, E. (2010). An augmented passive immune therapy to treat fulminant bacterial infections. Recent Pat Antiinfect Drug Discov, 5(2), 157-167.

[7] Virdi, V., \& Depicker, A. (2013). Role of plant expression systems in antibody production for passive immunization. Int J Dev Biol, 57(6-7-8), 587-593.

[8] Burnouf, T., \& Seghatchian, J. (2014). Ebola virus convalescent blood products: where we are now and where we may need to go. Transfus Apher Sci, 51(2), 120-125.

[9] Manohar, A., Ahuja, J., \& Crane, J. K. (2015). Immunotherapy for infectious diseases: Past, present, and future. Immunol Invest, 44(8), 731-737.

[10] Agency, A. (2020). Convalescent plasma therapy useful in treating COVID-19. Available online: https://www.aa.com.tr/en/health/convalescent-plasma-therapy-useful-in-treating-covid-19/1797975

[11] Stöppler, M. C. (2020). Apheresis (Hemapheresis, Pheresis). Available online: https://www.medicinenet.com/hemapheresis/article.htm\#what_are_some_possible_complications_of_apheresis

[12] Dodd, R. Y. (2012). Emerging pathogens and their implications for the blood supply and transfusion transmitted infections. Br J Haematol, 159(2), 135-142.

[13] Luke, T. C., Casadevall, A., Watowich, S. J., Hoffman, S. L., Beigel, J. H., \& Burgess, T. H. (2010). Hark back: passive immunotherapy for influenza and other serious infections. Crit Care Med, 38, e66-e73.

[14] Bogardus, F. (1919). Influenza Pneumonia Treated by Blood Transfusion. NY Med J, 12(6).

[15] Carlyle, P. (1919). Injection of whole blood in influenza. Br Med J, 1, 698.

[16] Francis, F., Hall, M., \& Gaines, A. (1920). Early use of convalescent serum in influenza. Mil Surg, 47, 177-179.

[17] Lakartidnin, S. (1920). Treatment of influenza pneumonia with serum from convalescents. Svenska Lakartidnin, 18, 385-399.

[18] Miller, O., \& McConnell, W. (1919). Report of influenza treated with serum from recovered cases. Ky Med J, 17, $218-219$.

[19] Redden, W. R. (1919). Treatment of influenza-pneumonia by use of convalescent human serum. Boston Med Surg J, 181(24), 688-691.

[20] Bakr Nour, M. G., Michaels, M., Reyes, J., Tzakis, A., Gartner, J. C., McLoughlin, L., \& Starzl, T. E. (1993). Parvovirus B19 infection in pediatric transplant patients. Transplantation, 56(4), 835.

[21] Beigel, J. H., \& Luke, T. C. (2012). A study in scarlet-convalescent plasma for severe influenza. Crit Care Med, $40(3), 1027$.

[22] Group, W. M.-C. R. (2013). State of knowledge and data gaps of Middle East respiratory syndrome coronavirus (MERS-CoV) in humans. PLoS Curr, 5.

[23] Hemming, V. G. (2001). Use of intravenous immunoglobulins for prophylaxis or treatment of infectious diseases. Clin Diagn Lab Immunol, 8(5), 859-863.

[24] Hui, D. S., \& Lee, N. (2013). Adjunctive therapies and immunomodulating agents for severe influenza. Influenza Other Resp Viruses, 7, 52-59.

[25] Kong, L., \& Zhou, B. (2006). Successful treatment of avian influenza with convalescent plasma. Hong Kong Med J, 12(6), 489.

[26] Leider, J. P., Brunker, P. A., \& Ness, P. M. (2010). Convalescent transfusion for pandemic influenza: preparing blood banks for a new plasma product? Transfusion, 50(6), 1384-1398. 
[27] Young, M. K., Nimmo, G. R., Cripps, A. W., \& Jones, M. A. (2014). Post - exposure passive immunisation for preventing measles. Cochrane Database Syst Rev(4).

[28] Zhou, B., Zhong, N., \& Guan, Y. (2007). Treatment with convalescent plasma for influenza A (H5N1) infection. N Engl J Med, 357(14), 1450-1451.

[29] Zingher, A., \& Mortimer, P. (2005). Convalescent whole blood, plasma and serum in the prophylaxis of measles. Rev Med Virol, 15(6), 407.

[30] Wong, V., Dai, D., Wu, A., \& Sung, J. (2003). Treatment of severe acute respiratory syndrome with convalescent plasma. Hong Kong Med J, 9(3), 199-201.

[31] Mozdzanowska, K., Furchner, M., Washko, G., Mozdzanowski, J., \& Gerhard, W. (1997). A pulmonary influenza virus infection in SCID mice can be cured by treatment with hemagglutinin-specific antibodies that display very low virusneutralizing activity in vitro. J Virol, 71(6), 4347-4355.

[32] Prince, G. A., Hemming, V. G., Horswood, R. L., Baron, P. A., \& Chanock, R. M. (1987). Effectiveness of topically administered neutralizing antibodies in experimental immunotherapy of respiratory syncytial virus infection in cotton rats. $\mathrm{J}$ Virol, 61(6), 1851-1854.

[33] Simmons, C. P., Bernasconi, N. L., Suguitan Jr, A. L., Mills, K., Ward, J. M., Chau, N. V. V., . . . Farrar, J. (2007). Prophylactic and therapeutic efficacy of human monoclonal antibodies against H5N1 influenza. PLoS Med, 4(5).

[34] McGuire, L., \& Redden, W. (1918). The use of convalescent human serum in influenza pneumonia-a preliminary report. Am J Public Health, 8(10), 741.

[35] Gunn, W. (1932). Convalescent serum in prophylaxis of measles, chicken-pox, and mumps. Br Med J, 1(3708), 183.

[36] Gundersen, T. (1935). Convalescent blood for herpes zoster. Trans Am Ophthalmol Soc, 33, 508.

[37] Jensen, C. (1935). The 1934 epidemic of poliomyelitis in Denmark. Preliminary report on the epidemiology, clinical features and convalescent serum therapy. Proc R Soc Med, 28(8), 1007-1026.

[38] Nabarro, D., \& Signy, A. (1931). Convalescent serum in prophylaxis of measles. Br Med J, 1(3652), 12.

[39] Organization, W. H. (2014a). Experimental therapies: growing interest in the use of whole blood or plasma from recovered Ebola patients (convalescent therapies). Media centre. Available online: https://www.who.int/mediacentre/news/ebola/26september-2014/en/

[40] Organization, W. H. (2014b). Use of convalescent whole blood or plasma collected from patients recovered from Ebola virus disease for transfusion, as an empirical treatment during outbreaks: interim guidance for national health authorities and blood transfusion services.

[41] Winkler AM, \& SA, K. (2015). The use of convalescent plasma to treat emerging infectious diseases: focus on Ebola virus disease. Curr Opin Hematol, 22(6), 521-526.

[42] Enria, D., Fernandez, N., Briggiler, A., Levis, S., \& Maiztegui, J. (1984). Importance of dose of neutralising antibodies in treatment of Argentine haemorrhagic fever with immune plasma. Lancet, 324(8397), 255-256.

[43] Enria, D. A., Briggiler, A. M., \& Sánchez, Z. (2008). Treatment of Argentine hemorrhagic fever. Antivir Res, 78(1), $132-139$.

[44] Enria, D. A., \& Maiztegui, J. I. (1994). Antiviral treatment of Argentine hemorrhagic fever. Antivir Res, 23(1), 23-31.

[45] Maiztegui, J., Fernandez, N., \& De Damilano, A. (1979). Efficacy of immune plasma in treatment of Argentine haemorrhagic fever and association between treatment and a late neurological syndrome. Lancet, 314(8154), 1216-1217.

[46] Ruggiero, H., Pérez, F. I., Milani, H., Barri, A., Val, A., Maglio, F., . . . Tallone, J. (1986). Treatment of Argentine hemorrhagic fever with convalescent's plasma. 4433 cases. Presse Med, 15(45), 2239-2242.

[47] Jahrling, P. B., Frame, J. D., Rhoderick, J. B., \& Monson, M. H. (1985). Endemic Lassa fever in Liberia. IV. Selection of optimally effective plasma for treatment by passive immunization. Trans R Soc Trop Med Hyg, 79(3), 380-384.

[48] Organization, W. H. (2003a). Consensus document on the epidemiology of severe acute respiratory syndrome (SARS).

[49] Organization, W. H. (2003c). Summary of probable SARS cases with onset of illness from 1 November 2002 to 31 July 2003. Available online: https://www.who.int/csr/sars/country/table2004_04_21/en/

[50] Organization, W. H. (2003b). Severe acute respiratory syndrome (SARS): report by the Secretariat. Geneva: WHO Executive Board. EB113/33. Available online: https://apps.who.int/iris/handle/10665/20038

[51] Cheng, Y., Wong, R., Soo, Y., Wong, W., Lee, C., Ng, M., . . Cheng, G. (2005). Use of convalescent plasma therapy in SARS patients in Hong Kong. Eur J Clin Microbiol Infect Dis, 24(1), 44-46. 
[52] Yeh, K.-M., Chiueh, T.-S., Siu, L., Lin, J.-C., Chan, P. K., Peng, M.-Y., . . . Perng, C.-L. (2005). Experience of using convalescent plasma for severe acute respiratory syndrome among healthcare workers in a Taiwan hospital. J Antimicrob Chemother, 56(5), 919-922.

[53] Wong, V. W., Dai, D., Wu, A. K., \& Sung, J. J. (2003). Treatment of severe acute respiratory syndrome with convalescent plasma. Hong Kong Med J, 9(3), 199-201.

[54] Kong, L. (2003). Severe acute respiratory syndrome (SARS). Transfus Apher Sci, 29(1), 101. doi:10.1016/s14730502(03)00109-5.

[55] Nie, Q. H., Luo, X. D., \& Hui, W. L. (2003). Advances in clinical diagnosis and treatment of severe acute respiratory syndrome. World J Gastroenterol, 9(6), 1139-1143. doi:10.3748/wjg.v9.i6.1139.

[56] Alraddadi, B. M., Watson, J. T., Almarashi, A., Abedi, G. R., Turkistani, A., Sadran, M., . . Madani, T. A. (2016). Risk Factors for Primary Middle East Respiratory Syndrome Coronavirus Illness in Humans, Saudi Arabia, 2014. Emerg Infect Dis, 22(1), 49-55. doi:10.3201/eid2201.151340.

[57] Organization, W. H. (2020b). Epidemic and pandemic-prone diseases. Available online: http://www.emro.who.int/pandemicepidemic-diseases/mers-cov/mers-situation-update-january-2020.html

[58] Chan, K. H., Chan, J. F., Tse, H., Chen, H., Lau, C. C., Cai, J. P., . . Y Yuen, K. Y. (2013). Cross-reactive antibodies in convalescent SARS patients' sera against the emerging novel human coronavirus EMC (2012) by both immunofluorescent and neutralizing antibody tests. J Infect, 67(2), 130-140. doi:10.1016/j.jinf.2013.03.015.

[59] Perera, R. A., Wang, P., Gomaa, M. R., El-Shesheny, R., Kandeil, A., Bagato, O., . . Kayali, G. (2013). Seroepidemiology for MERS coronavirus using microneutralisation and pseudoparticle virus neutralisation assays reveal a high prevalence of antibody in dromedary camels in Egypt, June 2013. Euro Surveill, 18(36), pii=20574. doi:10.2807/15607917.es2013.18.36.20574.

[60] Reusken, C. B., Haagmans, B. L., Müller, M. A., Gutierrez, C., Godeke, G. J., Meyer, B., . . Koopmans, M. P. (2013). Middle East respiratory syndrome coronavirus neutralising serum antibodies in dromedary camels: a comparative serological study. Lancet Infect Dis, 13(10), 859-866. doi:10.1016/s1473-3099(13)70164-6.

[61] Ying, T., Li, H., Lu, L., Dimitrov, D. S., \& Jiang, S. (2015). Development of human neutralizing monoclonal antibodies for prevention and therapy of MERS-CoV infections. Microbes Infect, 17(2), 142-148. doi:10.1016/j.micinf.2014.11.008

[62] Corti, D., Zhao, J., Pedotti, M., Simonelli, L., Agnihothram, S., Fett, C., . . . Lanzavecchia, A. (2015). Prophylactic and postexposure efficacy of a potent human monoclonal antibody against MERS coronavirus. Proc Natl Acad Sci U S A, 112(33), 10473-10478. doi:10.1073/pnas.1510199112.

[63] Ko, J. H., Seok, H., Cho, S. Y., Ha, Y. E., Baek, J. Y., Kim, S. H., . . Peck, K. R. (2018). Challenges of convalescent plasma infusion therapy in Middle East respiratory coronavirus infection: a single centre experience. Antivir Ther, 23(7), 617-622. doi:10.3851/imp3243.

[64] Ko, J. H., Müller, M. A., Seok, H., Park, G. E., Lee, J. Y., Cho, S. Y., . . Peck, K. R. (2017a). Serologic responses of 42 MERS-coronavirus-infected patients according to the disease severity. Diagn Microbiol Infect Dis, 89(2), 106-111. doi:10.1016/j.diagmicrobio.2017.07.006.

[65] Ko, J. H., Park, G. E., Lee, J. Y., Lee, J. Y., Cho, S. Y., Ha, Y. E., . . Peck, K. R. (2016). Predictive factors for pneumonia development and progression to respiratory failure in MERS-CoV infected patients. J Infect, 73(5), 468-475. doi:10.1016/j.jinf.2016.08.005.

[66] Ko, J. H., Müller, M. A., Seok, H., Park, G. E., Lee, J. Y., Cho, S. Y., . . Peck, K. R. (2017b). Suggested new breakpoints of anti-MERS-CoV antibody ELISA titers: performance analysis of serologic tests. Eur J Clin Microbiol Infect Dis, 36(11), 2179-2186. doi:10.1007/s10096-017-3043-3.

[67] Arabi, Y. M., Hajeer, A. H., Luke, T., Raviprakash, K., Balkhy, H., Johani, S., . . Alahmadi, M. (2016). Feasibility of Using Convalescent Plasma Immunotherapy for MERS-CoV Infection, Saudi Arabia. Emerg Infect Dis, 22(9), $1554-1561$. doi:10.3201/eid2209.151164.

[68] Mair-Jenkins, J., Saavedra-Campos, M., Baillie, J. K., Cleary, P., Khaw, F. M., Lim, W. S., . . . Beck, C. R. (2015). The effectiveness of convalescent plasma and hyperimmune immunoglobulin for the treatment of severe acute respiratory infections of viral etiology: a systematic review and exploratory meta-analysis. J Infect Dis, 211(1), 80-90. doi:10.1093/infdis/jiu396.

[69] Arabi, Y., Balkhy, H., Hajeer, A. H., Bouchama, A., Hayden, F. G., Al-Omari, A., . . Fowler, R. (2015). Feasibility, safety, clinical, and laboratory effects of convalescent plasma therapy for patients with Middle East respiratory syndrome coronavirus infection: a study protocol. Springerplus, 4, 709. doi:10.1186/s40064-015-1490-9. 
[70] Casadevall, A., \& Pirofski, L. A. (2020). The convalescent sera option for containing COVID-19. J Clin Invest, 130(4), 15451548. doi:10.1172/jci138003.

[71] Kraft, C. S., Hewlett, A. L., Koepsell, S., Winkler, A. M., Kratochvil, C. J., Larson, L., . . Ribner, B. S. (2015). The Use of TKM-100802 and Convalescent Plasma in 2 Patients with Ebola Virus Disease in the United States. Clin Infect Dis, 61(4), 496-502. doi:10.1093/cid/civ334.

[72] Chen, L., Xiong, J., Bao, L., \& Shi, Y. (2020). Convalescent plasma as a potential therapy for COVID-19. Lancet Infect Dis, 20(4), 398.

[73] Zhang, L., \& Liu, Y. (2020). Potential interventions for novel coronavirus in China: A systematic review. J Med Virol, 92(5), 479-490. doi:10.1002/jmv.25707.

[74] Organization, W. H. (2020a). Clinical management of severe acute respiratory infection (SARI) when COVID-19 disease is suspected: interim guidance, 13 March 2020.

[75] Xinhua. (2020). China puts 245 COVID-19 patients on convalescent plasma therapy. Available online: http://www.xinhuanet.com/english/2020-02/28/c_138828177.htm

[76] Duan, K., Liu, B., Li, C., Zhang, H., Yu, T., Qu, J., . . Hu, Y. (2020). Effectiveness of convalescent plasma therapy in severe COVID-19 patients. Proc Natl Acad Sci U S A, 117(17), 9490-9496.

[77] Food, U., \& Administration, D. (2020). Investigational COVID-19 Convalescent Plasma-Emergency INDs. Available online: http://natap.org/2020/COVID/032320_39.htm 